\title{
Hassalstrongylus dollfusi (Nematoda, Heligmonellidae): rediscovery in native South American rodents, six decades after its description
}

Paula Carolina Serrano ${ }^{1,2, *}$, María Celina Digiani ${ }^{1,2}$, María de los Angeles Gómez-Muñoz ${ }^{3}$, Juliana Notarnicola ${ }^{4}$ María del Rosario Robles ${ }^{5}$, and Graciela Teresa Navone ${ }^{5}$

${ }^{1}$ CONICET-Consejo Nacional de Investigaciones Científicas y Técnicas, Argentina

2 División Zoología Invertebrados, Facultad de Ciencias Naturales y Museo, Universidad Nacional de La Plata, Paseo del Bosque s/n, 1900 La Plata, Argentina

${ }^{3}$ Laboratorio Biología de los Parásitos, Facultad de Ciencias Exactas y Naturales y Agrimensura, Universidad Nacional del Nordeste, Av. Libertad 5460, 3400 Corrientes, Argentina

${ }^{4}$ Instituto de Biología Subtropical (CONICET - Universidad Nacional de Misiones), Bertoni 85, 3370 Puerto Iguazú, Argentina

${ }^{5}$ Centro de Estudios Parasitológicos y de Vectores (CCT La Plata-CONICET-UNLP), 120 e/61 y 62, B1900FWA La Plata, Argentina

Received 27 May 2021, Accepted 16 November 2021, Published online 9 December 2021

\begin{abstract}
Hassalstrongylus dollfusi (Díaz-Ungría, 1963) Durette-Desset, 1971 was described in a wild house mouse, Mus musculus, from Venezuela and, since then, has never been reported again in the type host or in any other host. In this work, specimens assignable to $H$. dollfusi were found at 10 localities in Northeast Argentina, in five species of sigmodontine rodents. The nematodes were attributed to $H$. dollfusi based on diagnostic characters such as: synlophe with 22-31 subequal ridges; in males, hypertrophy of right ray 4 of the male bursa, thickening of the dorsal ray and bases of rays 8, distal tip of the spicules bent and spoon shaped; and, in females, presence of subventral postvulvar alae supported by hypertrophied struts. The new host recorded are: Oligoryzomys fornesi, O. flavescens, O. nigripes, Holochilus chacarius and Akodon azarae. The parasite showed a strong preference for host species of Oligoryzomys, which appear to act as primary hosts. The parasite could be present, parasitizing different species of Oligoryzomys, in a geographic area from the type locality in Venezuela southward to north Corrientes in Argentina. It has not been reported from populations of Oligoryzomys spp. of the Argentinean and Brazilian Atlantic Forest, nor south of $28^{\circ} \mathrm{S}$, which may be explained by constraints in the environmental conditions required by the free-living stages of the parasite. This study provides the first identification and redescription of $H$. dollfusi in southern South America, from autochthonous hosts, six decades after its description.
\end{abstract}

Key words: Nippostrongylinae, Helminths, Sigmodontinae, Primary hosts, Northeast Argentina, Venezuela.

Résumé - Hassalstrongylus dollfusi (Nematoda, Heligmonellidae) : redécouverte chez des rongeurs autochtones d'Amérique du Sud, six décennies après sa description. Hassalstrongylus dollfusi (Díaz-Ungría, 1963) DuretteDesset, 1971 a été décrit chez une souris grise sauvage, Mus musculus, au Venezuela et, depuis lors, n'a plus jamais été signalé chez l'hôte-type ni chez aucun autre hôte. Dans ce travail, des spécimens attribuables à $H$. dollfusi ont été trouvés dans dix localités du nord-est de l'Argentine, chez cinq espèces de rongeurs Sigmodontinae. Les nématodes ont été attribués à $H$. dollfusi sur la base de caractères diagnostiques tels que : synlophe avec 22-31 crêtes subégales; chez le mâle, hypertrophie de la côte 4 droite de la bourse, épaississement de la côte dorsale et des bases des côtes 8, extrémité distale des spicules recourbée et en forme de cuillère; et, chez les femelles, présence d'ailes postvulvaires subventrales à fort support cuticulaire. Les nouveaux hôtes signalés sont : Oligoryzomys fornesi, O. flavescens, O. nigripes, Holochilus chacarius et Akodon azarae. Le parasite a montré une forte préférence pour les espèces hôtes du genre Oligoryzomys, qui semblent agir comme hôtes primaires. Le parasite pourrait être présent, parasitant différentes espèces d'Oligoryzomys, dans une zone géographique allant de la localité-type au Vénézuéla vers le sud jusqu'au nord de Corrientes en Argentine. Il n’a pas été signalé dans les populations d'Oligoryzomys spp. de la Forêt Atlantique argentine et brésilienne, ni au sud de $28^{\circ} \mathrm{S}$, ce qui peut s'expliquer par des contraintes dans les conditions environnementales requises par les stades libres du parasite. Cette étude fournit la première identification et redescription d'H. dollfusi dans le sud de l'Amérique du Sud, à partir d'hôtes autochtones, six décennies après sa description.

*Corresponding author: pserrano@fcnym. unlp. edu. ar; caroserrano297@gmail.com 


\section{Introduction}

The Heligmonellidae are the most speciose family of the Trichostrongylina (Strongylida or bursate nematodes). Heligmonellids are distributed worldwide and are overwhelmingly parasites of rodents (ca. 350 species); very few species have been described from moles, lagomorphs, tragulids and dermopterans $(<20)$ [3, 22]. Among the five subfamilies of heligmonellids, the Nippostrongylinae include the largest number of species (ca. 230); nippostrongylines are widespread all over the world and occur mainly in the superfamily Muroidea, frequently with more than one species per host [22].

In the Americas, nippostrongylines are parasitic in the Cricetidae (Muroidea), which are represented by four subfamilies: the Arvicolinae (with Holarctic distribution), the Neotominae and Tylomyinae (North or Middle American lineages); and the highly diverse Sigmodontinae (autochthonous to South America) [36]. Together, these subfamilies include ca. 650 species of rodents [43]. In contrast, less than $10 \%$ of these host species (ca. 60) have been investigated for nippostrongylines, which are known to be a highly diverse group [13].

In addition to native cricetids, synanthropic species of rats and mice: Mus musculus Linnaeus, Rattus norvegicus Berkenhout and Rattus rattus Linnaeus (Muridae, Murinae) also act as hosts to nippostrongylines. Due to their importance in public health, the parasitic fauna of these rodents has been much more extensively surveyed ([1, 11, 24-27, 30, 34, 35] among the most recent). Both species of Rattus mentioned above harbour throughout their distribution their own species of nippostrongyline: Nippostrongylus brasiliensis (Travassos, 1914) [9, 40]. However, they can occasionally be parasitized with other nippostrongylines from autochthonous rodents $[14,20,35]$. In a similar way, domestic mice, though they are not usually parasitized by nippostrongylines, may become occasionally infected with $N$. brasiliensis $[9,34]$ and with nippostrongylines from native rodents $[12,14,23,35]$.

Among New World nippostrongylines, the genus Hassalstrongylus Durette-Desset, 1971 [16] includes 17 species distributed from the southern USA to central Argentina. Fourteen out of the 17 species parasitize only native sigmodontine hosts; however, Hassalstrongylus aduncus (Chandler, 1932), primarily parasitic in Sigmodontinae and Arvicolinae [4, 10, 17, 23], has also been reported in rats [20, 35] and Hassalstrongylus musculi (Dikmans, 1935), although described originally in mice [14], was later found repeatedly parasitizing several species of sigmodontines [29, 35, 41].

Hassalstrongylus dollfusi (Díaz-Ungría, 1963) was originally described as Longistriata dollfusi Díaz-Ungría, 1963 in a wild Mus musculus from Venezuela [12]. Some years later, Durette-Desset (1969) [15] described the synlophe on reexamination of the type material, and finally Durette-Desset (1971) [16] transferred the species to the genus Hassalstrongylus. These three contributions are the only existing publications concerning $H$. dollfusi, there having been no new records since its description in 1963. It was, up to now, the only species of Hassalstrongylus reported exclusively from an exotic Murinae.

In this work, we report the finding of $H$. dollfusi in five species of sigmodontine hosts in 10 different localities of Northeast Argentina, providing a complete morphological description and enlarging the range of morphometrical data on this species. Additionally, we provide ecological parameters for the different surveyed populations and discuss the status of the different host-parasite associations.

\section{Materials and methods \\ Ethics}

The research was performed in agreement with Argentine laws. The specimens, obtained using methods for live capture, were sacrificed following the procedures and protocols suggested by AVMA Guidelines on Euthanasia and approved by National Laws (Animal Protection National Law 14.346 and references in the provincial permits) and by the Ethics Committee for Research on Laboratory Animals, Farm and Obtained from Nature of the National Council of Scientific and Technical Research (CONICET). No endangered species were involved in this study.

\section{Materials}

In the framework of a more comprehensive project involving the study of the taxonomy, host and geographical distribution of different groups of helminths of sigmodontine rodents in Argentina, viscera of 580 rodents belonging to 13 species were examined for helminths: Akodon azarae (J. B. Fischer), $n=106$; Akodon montensis Thomas, $n=94$; Calomys callidus (Thomas), $n=4$; Calomys callosus (Rengger), $n=16$; Holochilus chacarius Thomas, $n=50$; Holochilus vulpinus (Brants), $n=9$; Necromys lasiurus (Lund), $n=77$; Necromys obscurus (Waterhouse), $n=10 ;$ Oligoryzomys flavescens (Waterhouse), $n=38$; Oligoryzomys fornesi (Massoia), $n=14$; Oligoryzomys nigripes (Olfers), $n=99$; Oxymycterus rufus (G. Fischer), $n=56$ and Sooretamys angouya (G. Fischer), $n=7$. Rodents were captured during several field studies involving various collaborators (see Acknowledgements) between 1999 and 2018 from a total of 29 localities distributed in seven provinces. The results presented herein refer only to the rodent species harbouring $H$. dollfusi (Fig. 1).

\section{Methods}

Nematodes were recovered by observation of the gastrointestinal tracts under a stereomicroscope, subsequently fixed in $4 \%$ formalin and preserved in $70 \%$ ethanol. Nippostrongylines were studied in temporary mounts in Amman's lactophenol under a Leica DM 2500 microscope provided with a drawing attachment. Photographs were taken with a Leica DMC5400 camera. The synlophe was studied following Durette-Desset [18] and the nomenclature referring to the axis of orientation of the ridges follows Durette-Desset and Digiani [19]. Ridges are numbered from left to right and with respect to the oblique axis of orientation: 1 to $\mathrm{n}$ for right-dorsal ridges, and $1^{\prime}$ to $\mathrm{n}^{\prime}$ for left-ventral ridges. The nomenclature used for the study of the bursa (pattern of lateral lobes and symmetry) follows DuretteDesset et al. [21]. Measurements, unless otherwise stated, are provided in micrometres as the range followed by the mean 


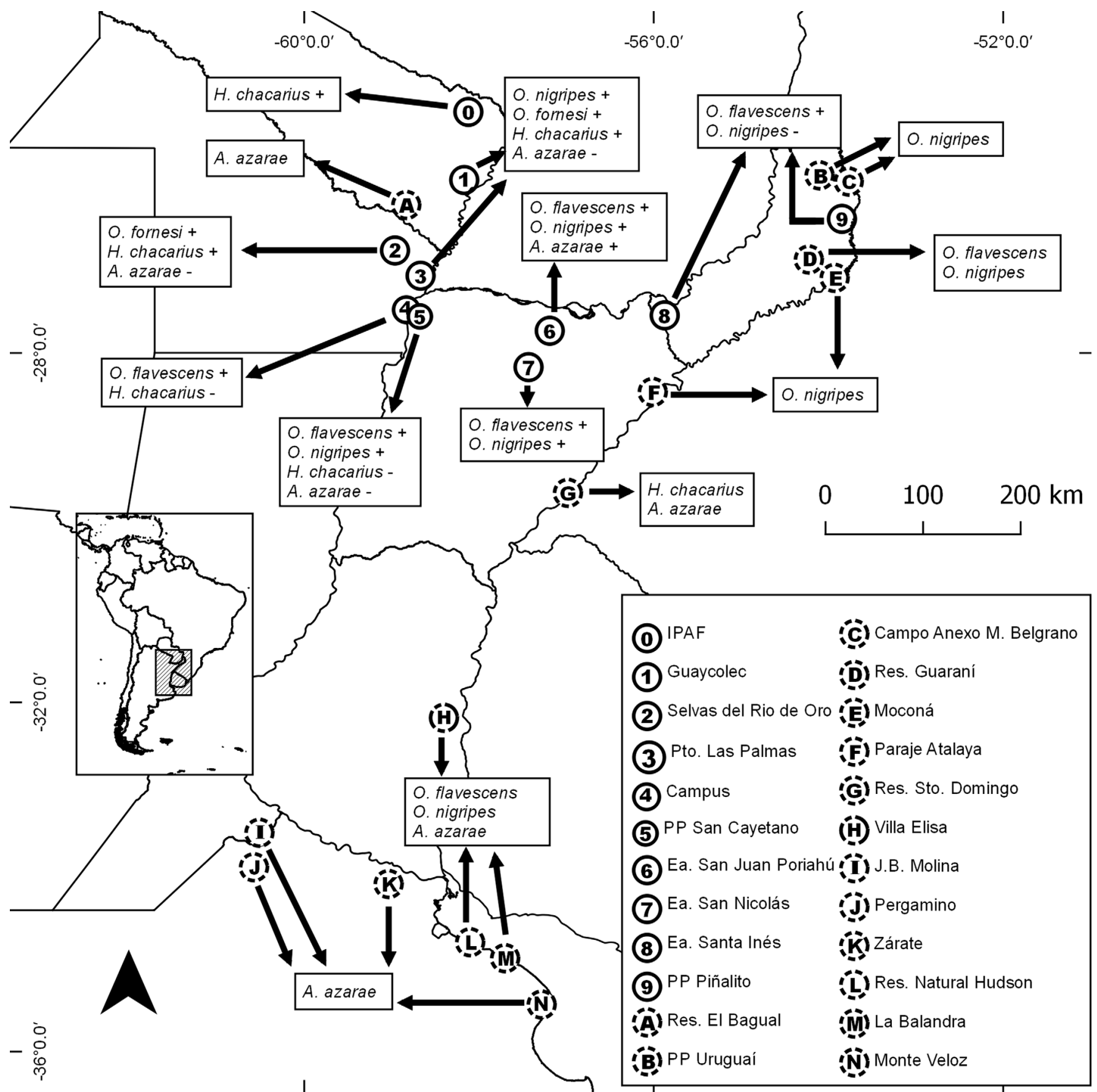

Figure 1. Sampled localities, including presence (0-9) and absence (A-N) of Hassalstrongylus dollfusi. Plus and minus symbols indicate the presence and absence of the parasite in each host, respectively. Localities B and C taken from Panisse [32]; Localities L and M taken from Navone et al. [31].

in parentheses. SpL/BL and UtL/BL mean the proportion of the spicule length to the body length and of the uterus length to the body length, respectively. EP/OeL means the position of the excretory pore (in percentage) with respect to the oesophagus length. Ecological parameters (prevalence and mean intensity) were calculated according to Bush et al. [5]. With the aim of evaluating the status of the different hosts harbouring $H$. dollfusi, the relative dominance (RD) of the latter in relation to the other intestinal heligmonellid species was calculated for each host species and locality. Figure 1 was constructed with free and open code software QGIS 2.14 [37].

Voucher specimens of helminths were deposited in the Helminthological Collection of the Museo de La Plata, La Plata, Buenos Aires, Argentina (MLP-He). Voucher specimens of hosts were deposited in the Mammalogical Collection of the Museo de La Plata (MLP-Mz) and Mammalogical Collection of the Centro Nacional Patagónico-CENPAT-CCT-CONICET, Puerto Madryn, Chubut, Argentina (CNP). 


\section{Results}

\section{Hassalstrongylus dollfusi (Díaz-Ungría, 1963) Durette-Desset, 1971}

\section{(= Longistriata dollfusi Díaz-Ungría, 1963)}

Site of Infection: Small intestine.

New hosts recorded: Akodon azarae (Sigmodontinae, Akodontini), Holochilus chacarius, Oligoryzomys flavescens, O. fornesi, O. nigripes (Sigmodontinae, Oryzomyini).

Localities (Fig. 1, Table 1):

(0) Instituto de Investigación para la Pequeña Agricultura Familiar del Noreste Argentino (IPAF- NEA), Laguna Blanca, Departamento Pilcomayo, Formosa province $\left(25^{\circ} 12^{\prime} 09.91^{\prime \prime} \mathrm{S}, 58^{\circ} 07^{\prime} 13.71^{\prime \prime} \mathrm{W}\right.$ ) (IPAF) (only H. chacarius examined from this locality. Not included in Table 1);

(1) Estación de Animales Silvestres Guaycolec, Departamento Formosa, Formosa province $\left(25^{\circ} 58^{\prime} 57.08^{\prime \prime} \mathrm{S}\right.$, $\left.58^{\circ} 10^{\prime} 04.00^{\prime \prime} \mathrm{W}\right)(\mathrm{GUAY})$;

(2) Selvas del Río de Oro, Departamento Libertador General San Martín, Chaco province, $\left(26^{\circ} 47^{\prime} 23.35^{\prime \prime} \mathrm{S}\right.$, $\left.58^{\circ} 57^{\prime} 37.81^{\prime \prime} \mathrm{W}\right)(\mathrm{SRDO})$;

(3) Puerto Las Palmas, Departamento Bermejo, Chaco province, three sampling points $\left(27^{\circ} 04^{\prime} 45.00^{\prime \prime} \mathrm{S}\right.$, $\left.58^{\circ} 40^{\prime} 06.30^{\prime \prime} \mathrm{W}\right) ; 7 \mathrm{~km} \quad \mathrm{~S}$ Puerto Las Palmas $\left(27^{\circ} 09^{\prime} 40.51^{\prime \prime} \mathrm{S}, 58^{\circ} 40^{\prime} 27.29^{\prime \prime} \mathrm{W}\right) ; 2.5 \mathrm{~km}$ NW Puerto Las Palmas $\left(27^{\circ} 04^{\prime} 45.00^{\prime \prime}\right.$ 'S, 58 $\left.40^{\circ} 06.30^{\prime \prime} \mathrm{W}\right)$ (PULP);

(4) Campus "Deodoro Roca"-Universidad Nacional del Nordeste, Departamento Capital, Corrientes province (2728 $09.17^{\prime \prime} \mathrm{S}, 58^{\circ} 49^{\prime} 50.29^{\prime \prime} \mathrm{W}$ ) (CAMP);

(5) Parque Provincial San Cayetano, Departamento Capital, Corrientes province, two sampling points $\left(27^{\circ} 32^{\prime} 43.88^{\prime \prime} \mathrm{S}\right.$, $\left.58^{\circ} 40^{\prime} 33.73^{\prime \prime} \mathrm{W}\right) ;\left(27^{\circ} 34^{\prime} 15^{\prime \prime} \mathrm{S} ; 58^{\circ} 41^{\prime} 41^{\prime \prime} \mathrm{W}\right)$ (SCAY);

(6) Estancia San Juan Poriahú, Departamento San Miguel, Corrientes province $\left(27^{\circ} 42^{\prime} 34.09^{\prime \prime} \mathrm{S}, 57^{\circ} 11^{\prime} 19.08^{\prime \prime} \mathrm{W}\right)$ (SJPO);

(7) Estancia San Nicolás, 22 Km SE San Miguel, Departamento San Miguel, Corrientes province $\left(28^{\circ} 07^{\prime} 35.03^{\prime \prime} \mathrm{S}\right.$, $\left.57^{\circ} 25^{\prime} 53.73^{\prime \prime} \mathrm{W}\right)(\mathrm{SNIC})$;

(8) Estancia Santa Inés, RP 105, Km 8.5, Departamento Capital, Misiones province $\left(27^{\circ} 31^{\prime} 59^{\prime \prime} \mathrm{S}, 55^{\circ} 52^{\prime} 22.03^{\prime \prime} \mathrm{W}\right.$ ) (SINE);

(9) Parque Provincial Piñalito, Departamento San Pedro, Misiones province $\left(26^{\circ} 25^{\prime} 40.07^{\prime \prime} \mathrm{S}, 53^{\circ} 50^{\prime} 38.26^{\prime \prime} \mathrm{W}\right)$ (PPPI).

\section{Prevalence and mean intensity: see Table 1.} Material studied and deposited: see Table 2

\section{Redescription (Figs. 2-6, Table 3)}

General: medium-sized nematodes, generally uncoiled, sometimes loosely coiled in 1-3 spirals. Cephalic vesicle present. Excretory pore always within posterior half of oesophagus (Table 3). Deirids at same level of excretory pore in most specimens ( $74 \%$ of 50 specimens), sometimes 3-25 $\mu \mathrm{m}$ anterior or posterior to it (Fig. 2A).

Head: in apical view, triangular buccal opening surrounded by thick ring; 2 amphids, 4 externo-labial ( 2 dorsal, 2 ventral), and 4 cephalic papillae visible; lateral externo-labial papillae probably fused with amphids (Fig. 2B).

Synlophe (studied in 6 males and 6 females): in both sexes, cuticle bearing longitudinal, uninterrupted ridges with welldeveloped struts. Ridges appearing gradually mainly on left side just posterior to cephalic vesicle up to oesophago-intestinal junction; disappearing just anterior to bursa in male and reaching distal cuticular inflation in females. Number of ridges variable in proportion to body diameter: at oesophago-intestinal junction, 22-26 in both sexes (Figs. 2C, 2D); at midbody 22-31 in males, 22-30 in females (Figs. 2E, 2F); within posterior third of body length 24-26 in males, 23-28 in females (Figs. 2G, 2H). All ridges medium sized, except those on right ventral quadrant (smallest) and those associated with lateral hypodermal chords (slightly larger). At oesophago-intestinal junction, especially in male, difference in ridge size more marked than at midbody. At midbody, ridge $2^{\prime}$ largest. All ridges well oriented, determining a double axis of orientation of the ridges in both sexes: left axis inclined at $83^{\circ}-84^{\circ}$ with respect to sagittal axis, right axis at $70^{\circ}-72^{\circ}$. Within distal third of body length: in both sexes, similar number and orientation than at midbody, differences in ridge size less marked (Figs. 2G, 2H). In females, between vulva and anus, presence of paired ventral postvulvar alae supported by hypertrophied struts (Fig. 3A).

Males (measurements reported by host in Table 3): bursa slightly dissymmetrical, with right lobe larger than left (Figs. 3B, 5A). Prebursal papillae not observed. Right lobe with pattern of type 2-2-1 tending to 1-3-1. Left lobe with pattern of type 2-3 tending to 2-2-1. Rays 2 shorter than rays 3 and curved toward median line. Rays 4 and 5 diverging at extremity, rays 4 longer, strongly curved anteriad, rays 5 straight. Right ray 4 markedly thicker than left one. Right ray 6 diverging from common trunk of rays 2-6 between rays 2 and 3; left ray 6 diverging distally in different degrees to ray 3 : from just distal (Fig. $3 \mathrm{~B}_{2}$ ) up to halfway between ray 3 and divergence of rays 4 and $5\left(\right.$ Fig. $\left.3 \mathrm{~B}_{1}\right)$. Rays 8 thickened at base, arising symmetrically from base of dorsal ray. Dorsal ray short, thickened at base, dividing within middle third into two branches, each one bifurcated distally into two papillae, external rays 9 and internal rays 10 . Genital cone well developed. Telamon complex, composed of several sclerotized pieces: two dorsal simple branches forming a pincer or caliper, and two ventral bifid branches articulated distally (Fig. 4A). Gubernaculum inconspicuous, Spicules thin, subequal, alate. Each spicule tip spatulate or spoonshaped, bent at right angle with respect to main axis of spicule. Just before bending, spicular ala enlarged and ending widely, with the appearance of a heeled shoe (Figs. 4B and 5B, 5C).

Females (measurements reported by host in Table 3): reproductive tract monodelphic. Uterus less than $20 \%$ of BL, number of eggs variable (see Table 3). Infundibulum slightly longer than vestibule. Distal end, in most specimens, curved ventrally to different degrees, more rarely straight (on 50 females examined, 19\% strongly curved, 30\% curved at right angle, $28 \%$ slightly curved and $23 \%$ straight). Cuticle inflated to different degrees from level of sphincter or vestibule up to halfway between vulva and anus (Figs. 4C and 6). Two latero-ventral alae present between vulva and anus (Fig. 4C). Tail conical, not invaginated (Figs. 4C and 6). 
Table 1. Values of Prevalence (P), Mean Intensity (MI), total number of worms (TNW) and Relative Dominance (RD) for Hassalstrongylus dollfusi, separated by host and locality. Numbers in parentheses indicate the total number of intestinal heligmonellids in the respective component communities. (GUAY) Estación de Animales Silvestres Guaycolec, Formosa province; (SRDO) Selvas del Río de Oro, Chaco province; (PULP) Puerto Las Palmas, Chaco province; (CAMP) Campus "Deodoro Roca"-Universidad Nacional del Nordeste, Corrientes province; (SCAY) Parque Provincial San Cayetano, Corrientes province; (SJPO) Estancia San Juan Poriahú, Corrientes province; (SNIC) Estancia San Nicolás, Corrientes province; (SINE) Estancia Santa Inés, Misiones province; (PPPI) Parque Provincial Piñalito, Misiones province.

\begin{tabular}{|c|c|c|c|c|c|c|}
\hline & & Akodon azarae & Holochilus chacarius & Oligoryzomys flavescens & O. fornesi & O. nigripes \\
\hline \multirow[t]{5}{*}{ GUAY } & Host $n$ & 14 & 2 & 0 & 6 & 27 \\
\hline & $P(\%)$ & 0 & 50 & - & 100 & 66.7 \\
\hline & MI & 0 & 2 & - & 169.2 & 8.9 \\
\hline & TNW & $0(539)$ & $2(39)$ & - & 1015 (1066) & $160(541)$ \\
\hline & $\mathrm{RD}(\%)$ & 0 & 5.1 & - & 95.2 & 29.6 \\
\hline \multirow[t]{5}{*}{ SRDO } & Host $n$ & 1 & 29 & 0 & 7 & 0 \\
\hline & $P(\%)$ & 0 & 20.7 & - & 100 & - \\
\hline & MI & 0 & 2.2 & - & 12.7 & - \\
\hline & TNW & $0(158)$ & $13(3316)$ & - & 89 (139) & - \\
\hline & $\mathrm{RD}(\%)$ & 0 & 0.4 & - & 64 & - \\
\hline \multirow[t]{5}{*}{ PULP } & Host $n$ & 2 & 4 & 0 & 1 & 11 \\
\hline & $P(\%)$ & 0 & 25 & - & 100 & 27.3 \\
\hline & MI & 0 & 1 & - & 5 & 3.3 \\
\hline & TNW & $0(83)$ & $1(357)$ & - & $5(16)$ & $10(189)$ \\
\hline & RD (\%) & 0 & 0.3 & - & 31.2 & 5.3 \\
\hline \multirow[t]{5}{*}{ CAMP } & Host $n$ & 0 & 1 & 13 & 0 & 0 \\
\hline & $P(\%)$ & - & 0 & 92.3 & - & - \\
\hline & MI & - & 0 & 101.5 & - & - \\
\hline & TNW & - & $0(74)$ & $1218(1218)$ & - & - \\
\hline & $\mathrm{RD}(\%)$ & - & 0 & 100 & - & - \\
\hline \multirow[t]{5}{*}{ SCAY } & Host $n$ & 9 & 1 & 4 & 0 & 3 \\
\hline & $P(\%)$ & 0 & 0 & 50 & - & 33.3 \\
\hline & MI & 0 & 0 & 23 & - & 238 \\
\hline & TNW & $0(248)$ & $0(77)$ & $46(46)$ & - & $238(240)$ \\
\hline & $\mathrm{RD}(\%)$ & 0 & 0 & 100 & - & 99.2 \\
\hline \multirow[t]{5}{*}{ SJPO } & Host $n$ & 2 & 0 & 3 & 0 & 5 \\
\hline & $P(\%)$ & 50 & - & 100 & - & 40 \\
\hline & MI & 303 & - & 42.7 & - & 2 \\
\hline & TNW & $303(303)$ & - & $128(133)$ & - & $4(29)$ \\
\hline & $\mathrm{RD}(\%)$ & 100 & - & 96.2 & - & 13.8 \\
\hline \multirow[t]{5}{*}{ SNIC } & Host $n$ & 0 & 0 & 6 & 0 & 16 \\
\hline & $P(\%)$ & - & - & 100 & - & 75 \\
\hline & MI & - & - & 36.5 & - & 7.8 \\
\hline & TNW & - & - & 219 (219) & - & $93(93)$ \\
\hline & RD (\%) & - & - & 100 & - & 100 \\
\hline \multirow[t]{5}{*}{ SINE } & Host $n$ & 0 & 0 & 4 & 0 & 3 \\
\hline & $P(\%)$ & - & - & 75 & - & 0 \\
\hline & MI & - & - & 12.7 & - & 0 \\
\hline & TNW & - & - & $38(38)$ & - & $0(8)$ \\
\hline & RD (\%) & - & - & 100 & - & 0 \\
\hline \multirow[t]{5}{*}{ PPPI } & Host $n$ & 0 & 0 & 1 & 0 & 17 \\
\hline & $P(\%)$ & - & - & 100 & - & 0 \\
\hline & MI & - & - & 2 & - & 0 \\
\hline & TNW & - & - & $2(23)$ & - & 0 (499) \\
\hline & $\mathrm{RD}(\%)$ & - & - & 8.7 & - & 0 \\
\hline \multirow[t]{5}{*}{ Total } & Host $n$ & 28 & 37 & 31 & 14 & 82 \\
\hline & $P(\%)$ & 3.6 & 21.6 & 87.1 & 100 & 43.9 \\
\hline & MI & 303 & 2 & 61.1 & 79.2 & 14 \\
\hline & TNW & $303(1331)$ & $16(3867)$ & 1651 (1677) & 1109 (1221) & 505 (1361) \\
\hline & $\mathrm{RD}(\%)$ & 22.8 & 0.4 & 98.5 & 90.8 & 37.1 \\
\hline
\end{tabular}


Table 2. Examined material of Hassalstrongylus dollfusi. Acronyms for localities: IPAF: Instituto de Investigación para la Pequeña Agricultura Familiar del Noreste Argentino; GUAY: Estación de Animales Silvestres Guaycolec; SRDO: Selvas del Río de Oro; PULP: Puerto Las Palmas; CAMP: Campus "Deodoro Roca"-Universidad Nacional del Nordeste; SCAY: Parque Provincial San Cayetano; SJPO: Estancia San Juan Poriahú; SNIC: Estancia San Nicolás; SINE: Estancia Santa Inés; PPPI: Parque Provincial Piñalito. Acronyms for collections: CNP: Centro Nacional Patagónico - CCT - CONICET (Mammalogical Collection), Puerto Madryn, Argentina; MLP: Museo de La Plata (Mammalogical Collection), La Plata, Argentina; MLPHe: Museo de La Plata (Helminthological Collection), La Plata, Argentina.

\begin{tabular}{|c|c|c|c|c|c|c|c|c|c|}
\hline Province & Locality & Lat (S) & Long (W) & Year & Host species & Host collection No. & Host field No. & Infrapopulation & Nematodes collection No \\
\hline \multirow[t]{28}{*}{ Formosa } & IPAF & $\begin{array}{c}25^{\circ} \\
12^{\prime} 09.91^{\prime \prime}\end{array}$ & $\begin{array}{c}58^{\circ} \\
07^{\prime} 13.71^{\prime \prime}\end{array}$ & 2008 & $\begin{array}{l}\text { Holochilus } \\
\text { chacarius }\end{array}$ & & LTU 561 & $1 \hat{\sigma}^{\star}$ & MLP-He $7746(1 \hat{\jmath})$ \\
\hline & & & & & & CNP 2391 & LTU 565 & $1 \hat{\sigma}$ & \\
\hline & & & & & & CNP 3943 & LTU 571 & $1 \widehat{\widehat{\sigma}}$ & MLP-He $7746\left(1 \varsigma^{\wedge}\right)$ \\
\hline & GUAY & $\begin{array}{c}25^{\circ} \\
58^{\prime} 57.08^{\prime \prime}\end{array}$ & $\begin{array}{c}58^{\circ} \\
10^{\prime} 04.00^{\prime \prime}\end{array}$ & 2012 & $\begin{array}{c}\text { Oligoryzomys } \\
\text { nigripes }\end{array}$ & & CG 83 & $3 \hat{0}, 1$ 우 & 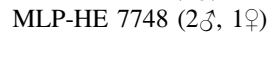 \\
\hline & & & & & & & CG 93 & $1 ㅇ$ & \\
\hline & & & & & & & CG 96 & $1 \hat{0}$ & MLP-HE $7748(1 \hat{\jmath})$ \\
\hline & & & & & & & CG 118 & 19 & \\
\hline & & & & & & & CG 125 & 1 우 & \\
\hline & & & & & & CNP 3877 & CG 133 & 1 우 & \\
\hline & & & & 2013 & H. chacarius & CNP 4775 & CG 448 & $2 \hat{\sigma}$ & \\
\hline & & & & & O. fornesi & CNP 5271 & CG 435 & $1 \widehat{\delta}, 1$ 우 & \\
\hline & & & & & & CNP 5010 & CG 444 & $25 \hat{\jmath}, 22$ 우 & \\
\hline & & & & & & & CG 445 & 35스, 42 우 & \\
\hline & & & & & & & CG 446 & $85 \hat{\jmath}, 105$ 우 & MLP-He 7753 (6今人, 7우) \\
\hline & & & & & & CNP 5277 & CG 449 & $230 \hat{\jmath}, 243$ 우 & \\
\hline & & & & & & & CG 453 & $101 \hat{\diamond}, 125$ 우 & MLP-He 7753 (1우) \\
\hline & & & & & O. nigripes & & CG 372 & 19 & \\
\hline & & & & & & CNP 4819 & CG 416 & $2 \hat{\sigma}$ & \\
\hline & & & & & & CNP 4736 & CG 418 & $5 \hat{\jmath}, 3$ 우 & \\
\hline & & & & & & & CG 420 & $1 \hat{0}$ & \\
\hline & & & & & & & CG 421 & $32 \hat{\jmath}, 61$ 우 & \\
\hline & & & & & & CNP 5073 & CG 422 & $4 \hat{\jmath}, 1+1+$ & \\
\hline & & & & & & CNP 5259 & CG 424 & $1 \hat{\delta}, 2$ 우 & \\
\hline & & & & & & & CG 426 & $4 \hat{\beta}, 10$ 우 & MLP-HE $7748(4 \hat{\sigma}, 4$ 우) \\
\hline & & & & & & & CG 427 & $3 \hat{\jmath}, 3+$ & \\
\hline & & & & & & & CG 428 & $1 \hat{\jmath}, 2$ 운 & \\
\hline & & & & & & & CG 438 & $1 \hat{\jmath}, 10$ 우 & \\
\hline & & & & & & CNP 5058 & CG 451 & $4 \hat{\jmath}, 19$ & \\
\hline \multirow[t]{16}{*}{ Chaco } & SRDO & $26^{\circ}$ & $58^{\circ}$ & 2000 & H. chacarius & & RORO 002 & $1 \widehat{0}$ & MLP-He 7747 (1今) \\
\hline & & $47^{\prime} 23.35^{\prime \prime}$ & $57^{\prime} 37.81^{\prime \prime}$ & & & & & & \\
\hline & & & & & & CNP 3956 & RORO 023 & $3 \hat{0}$ & MLP-He $7747\left(1 \sigma^{\wedge}\right)$ \\
\hline & & & & & & & RORO 033 & $3 \hat{0}$ & MLP-He 7747 (2§) \\
\hline & & & & & & CNP 3954 & RORO 035 & $10 \hat{~}$ & MLP-He pending $(1 \widehat{\jmath})$ \\
\hline & & & & & & CNP 3953 & RORO 056 & $1 \hat{\sigma}^{\hat{2}}$ & \\
\hline & & & & & & & RORO 058 & $3 \hat{0}$ & MLP-He 7747 (3ڤ̋) \\
\hline & & & & & & & RORO 060 & $2 \hat{\sigma}$ & MLP-He 7747 (2ふ) \\
\hline & & & & 2013 & O. fornesi & & CG 404 & $2 \hat{\sigma}$ & MLP-He $7754\left(23^{\prime}\right)$ \\
\hline & & & & & & & CG 405 & $8 \hat{0}, 9$ 우 & MLP-He $7754(2 \hat{\jmath}, 2$ 우 \\
\hline & & & & & & & CG 408 & $5 \hat{0}, 2$ 우 & \\
\hline & & & & & & & CG 409 & $26 \hat{\beta}, 23$ 우 & MLP-He 7754 (5ภิ, 3우) \\
\hline & & & & & & & CG 410 & $5 \hat{\jmath}, 7$ 우 & MLP-He 7754 (1乞̂) \\
\hline & & & & & & & CG 412 & 1 우 & \\
\hline & & & & & & & CG 413 & $10 \hat{~}$ & \\
\hline & PULP & $\begin{array}{c}27^{\circ} \\
04^{\prime} 45.00^{\prime \prime}\end{array}$ & $\begin{array}{c}58^{\circ} \\
40^{\prime} 06.30^{\prime \prime}\end{array}$ & 2008 & O. fornesi & & LTU 585 & $2 \hat{\jmath}, 3+$ & \\
\hline
\end{tabular}


Table 2. (Continued)

\begin{tabular}{|c|c|c|c|c|c|c|c|c|c|}
\hline Province & Locality & Lat (S) & Long (W) & Year & Host species & Host collection No. & Host field No. & Infrapopulation & Nematodes collection No. \\
\hline & $7 \mathrm{~km} \mathrm{~S}$ PULP & $27^{\circ} 09^{\prime} 40.51^{\prime \prime}$ & $58^{\circ} 40^{\prime} 27.29^{\prime \prime}$ & 2008 & $O$. nigripes & CNP 1748 & LTU 589 & 19 & \\
\hline & & & & & & CNP 5635 & LTU 593 & $2 \hat{3}, 4$ 우 & MLP-He $7749\left(1 \hat{\delta}^{\wedge}, 3\right.$ 우 $)$ \\
\hline & & & & & & CNP 5723 & LTU 595 & $2 \hat{\delta}, 1$ 우 & MLP-He $7749(2 \widehat{\jmath}, 1$ 우 $)$ \\
\hline & 2,5 km NW PULP & $27^{\circ} 04^{\prime} 45.00^{\prime \prime}$ & $58^{\circ} 40^{\prime} 06.30^{\prime \prime}$ & 2008 & H. chacarius & CNP 3937 & LTU 605 & 1 우 & MLP-He 7759 (1ㅇ) \\
\hline \multirow[t]{39}{*}{ Corrientes } & CAMP & $27^{\circ} 28^{\prime} 09.17^{\prime \prime}$ & $58^{\circ} 49^{\prime} 50.29^{\prime \prime}$ & 2013 & O. flavescens & & RO-197 & 75 & \\
\hline & & & & & & & RO-211 & 32 & \\
\hline & & & & & & & RO-216 & 39 & \\
\hline & & & & & & & RO-218 & 39 & \\
\hline & & & & & & & RO-219 & 17 & \\
\hline & & & & & & & RO-221 & 22 & \\
\hline & & & & 2014 & & & RO-347 & 108 & \\
\hline & & & & & & & RO-348 & 65 & \\
\hline & & & & & & & RO-352 & 230 & \\
\hline & & & & & & & RO-355 & 233 & \\
\hline & & & & & & & RO-358 & 301 & \\
\hline & & & & & & & RO-363 & 57 & \\
\hline & SCAY 1 & $27^{\circ} 32^{\prime} 43.88^{\prime \prime}$ & $58^{\circ} 40^{\prime} 33.73^{\prime \prime}$ & 2007 & O. flavescens & CNP 5617 & LTU 423 & 21수, 20 우 & MLP-He $7755(1 \widehat{\jmath}, 1$ 우) \\
\hline & & & & & & & LTU 440 & $3 \widehat{\delta}, 2$ 우 & \\
\hline & SCAY 2 & $27^{\circ} 34^{\prime} 15^{\prime \prime}$ & $58^{\circ} 41^{\prime} 41^{\prime \prime}$ & 2011 & $O$. nigripes & & RO-022 & 238 & MLP-He $7750(6 ð \hat{~}, 6$ 6 $)$ \\
\hline & SJPO & $27^{\circ} 42^{\prime} 34.09^{\prime \prime}$ & $57^{\circ} 11^{\prime} 19.08^{\prime \prime}$ & 2003 & Akodon azarae & MLP 18.III.02.11 & 211 & 127수, 176우 & MLP-He $7758(4 \hat{\jmath}, 7$ 우) \\
\hline & & & & & O. flavescens & MLP 17.XII.01.11 & 193 & $19 \hat{\jmath}, 17$ 우 & MLP-He $7756(3 \hat{\jmath}, 4$ 운 \\
\hline & & & & & $O$. nigripes & MLP 27.XII.01.8 & 209 & $1 \hat{\jmath}, 2+$ & MLP-He $7751(1 \widehat{\jmath}, 1$ 우 \\
\hline & & & & & & MLP 27.XII.01.9 & 213 & $1 \hat{\sigma}^{\hat{2}}$ & MLP-He $7751\left(1 \delta^{\curvearrowright}\right)$ \\
\hline & & & & 2007 & O. flavescens & & LTU 413 & 23추, 37우 & \\
\hline & & & & & & & LTU 416 & $14 \widehat{\delta}, 18$ 우 & \\
\hline & SNIC & $28^{\circ} 07^{\prime} 35.03^{\prime \prime}$ & $57^{\circ} 25^{\prime} 53.73^{\prime \prime}$ & 2010 & O. flavescens & CNP 5606 & UP 1070 & $1 \hat{\delta}, 2+$ & \\
\hline & & & & & & & UP 1083 & 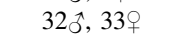 & \\
\hline & & & & & & & UP 1095 & $31 \widehat{\jmath}, 22$ + & \\
\hline & & & & & & & UP 1097 & $17 \hat{\delta}, 28$ ㅇ & \\
\hline & & & & & & CNP 5542 & UP 1098 & $8 \hat{3}, 16+$ & \\
\hline & & & & & & CNP 5631 & UP 1107 & 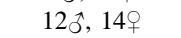 & \\
\hline & & & & & O. nigripes & & UP 1074 & $8 \hat{3}, 10+$ & MLP-He 7752 (1乞̂) \\
\hline & & & & & & CNP 5628 & UP 1076 & 19 & \\
\hline & & & & & & & UP 1077 & $18 \hat{\circ}, 11$ 우 & \\
\hline & & & & & & & UP 1080 & $1 \hat{0}, 4$ 우 & MLP-He 7752 (1우) \\
\hline & & & & & & CNP 5640 & UP 1081 & $3 \hat{0}, 1$ 우 & \\
\hline & & & & & & & UP 1088 & $1 \hat{\delta}, 4$ 우 & \\
\hline & & & & & & CNP 5603 & UP 1090 & 39 & \\
\hline & & & & & & & UP 1091 & $1 \hat{\delta}, 4$ 우 & \\
\hline & & & & & & & UP 1100 & $3 \hat{\delta}, 4$ 우 & \\
\hline & & & & & & CNP 5622 & UP 1101 & $4 \hat{6}, 3$ 우 & MLP-He $7752(2 \widehat{\jmath}, 2$ ㅇ $)$ \\
\hline & & & & & & CNP 5660 & UP 1102 & $3 \hat{\sigma}, 1$ 우 & MLP-He $7752(1 \widehat{\jmath}, 1$ 우 \\
\hline & & & & & & CNP 5611 & UP 1103 & $1 \hat{\delta}, 69$ & \\
\hline \multirow[t]{4}{*}{ Misiones } & SINE & $27^{\circ} 31^{\prime} 59^{\prime \prime}$ & $55^{\circ} 52^{\prime} 22.03^{\prime \prime}$ & 2009 & O. flavescens & & LTU 697 & $13 \hat{\delta}, 22$ 우 & MLP-He 7757 (5ภ̊, 6९) \\
\hline & & & & 2018 & O. flavescens & & CG 751 & $2 \hat{0}$ & \\
\hline & & & & & & & CG 775 & 1 우 & \\
\hline & PPPI & $26^{\circ} 25^{\prime} 40.07^{\prime \prime}$ & $53^{\circ} 50^{\prime} 38.26^{\prime \prime}$ & 2018 & O. flavescens & & CG 805 & 2 우 & \\
\hline
\end{tabular}



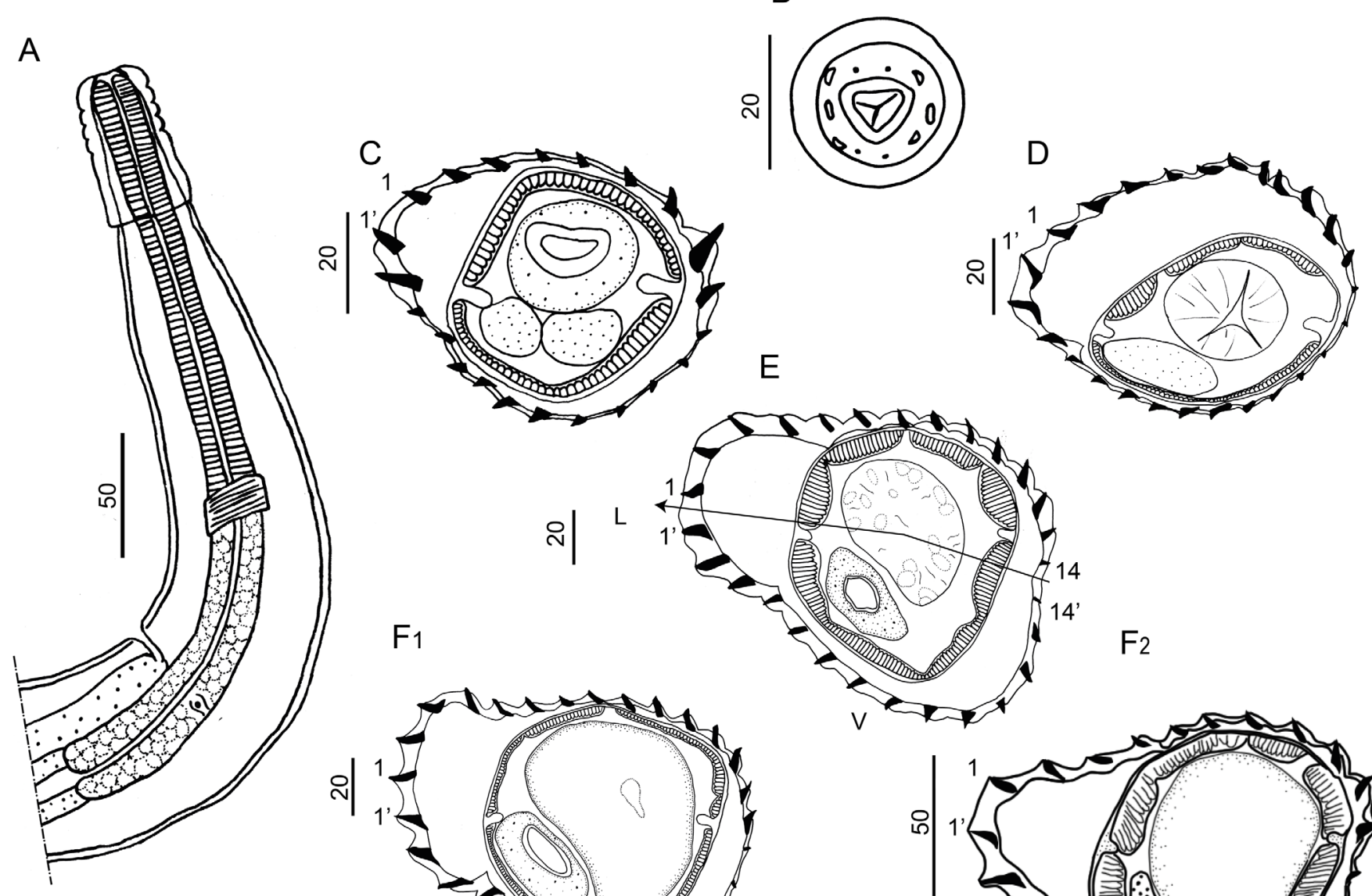

E

G

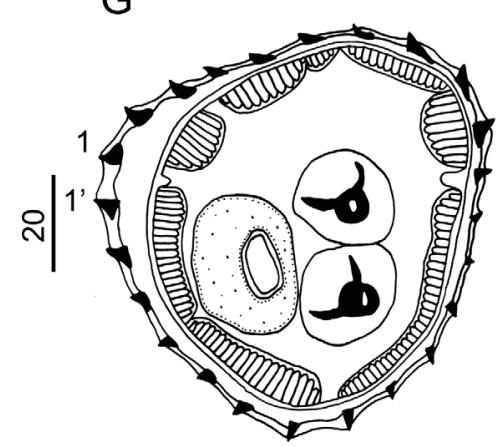

$\mathrm{H}$

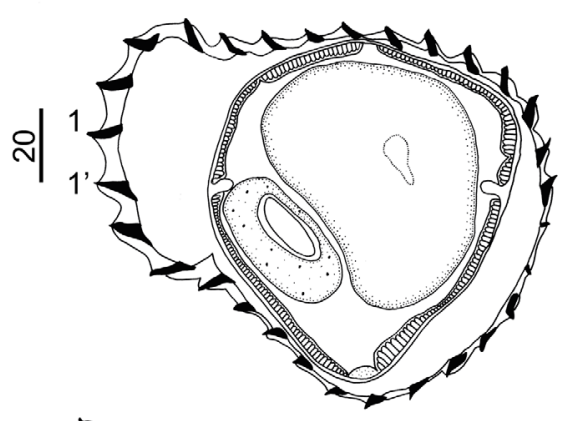

$\mathrm{F}_{2}$

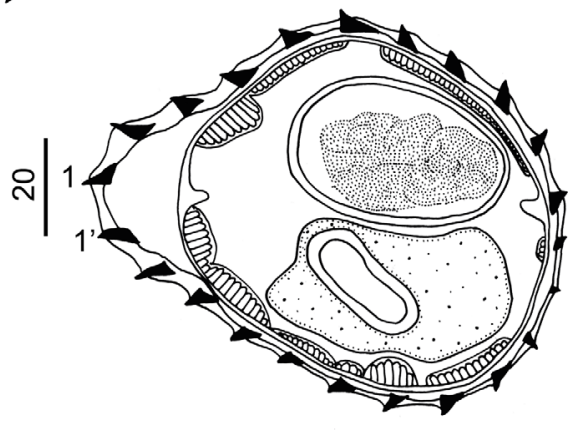

Figure 2. Hassalstrongylus dollfusi. A, male, anterior extremity, left lateral view. B, female, head, apical view. C-H, synlophe in transverse sections of the body: C, D, at oesophago-intestinal junction, C, male, D, female; E, F, at mid-body, E, male, F, female, (1) this work, (2) modified after Durette-Desset (1969); G, male, at level of spicules; H, female at level of uterus. Abbreviations: L, left, V, ventral. Arrow represents the axis of orientation of the ridges. All sections oriented as in E.

\section{Diagnosis}

Diagnostic characters of $H$. dollfusi males are: the morphology of the caudal bursa (particularly hypertrophy of right ray 4 and thickening of dorsal ray and rays 8 at their base), and the peculiar shape of the distal tip of the spicules. Diagnostic characters of the female are: the dorsal cuticular inflation at ovejector level and the subventral postvulvar alae supported by hypertrophied struts. These characters were present in all specimens studied herein and, compared to those in the original description [12] and further redescription [15], did not show any differences. In addition, the synlophes at midbody of males and females examined were congruent with those described by Durette-Desset [15]. Related to the measurements, they were homogenous among specimens from the different host species, including the type material from $M$. musculus. The specimens parasitizing $A$. azarae and $H$. chacarius were slightly smaller (Table 3).

Slight variability with respect to the original description was found only in two characters: the level of divergence of left ray 
Table 3. Comparative measurements of males and females of Hassalstrongylus dollfusi from the murid type host (from Díaz-Ungría 1963) and five native cricetid hosts (this work).

\begin{tabular}{|c|c|c|c|c|c|c|c|c|c|c|c|c|}
\hline & \multirow{2}{*}{\multicolumn{2}{|c|}{ Mus musculus }} & \multicolumn{2}{|c|}{ Akodon azarae } & \multicolumn{2}{|c|}{ Holochilus chacarius } & \multicolumn{2}{|c|}{ Oligoryzomys flavescens } & \multicolumn{2}{|c|}{ O. fornesi } & \multicolumn{2}{|c|}{ O. nigripes } \\
\hline & & & This & work & This work & & This $v$ & work & This & work & This v & work \\
\hline & $\begin{array}{c}\hat{0} \\
(n=\mathrm{np})\end{array}$ & $\begin{array}{c}+ \\
(n=\mathrm{np})\end{array}$ & $\widehat{\partial}(n=10)$ & $\uparrow(n=7)$ & $\widehat{\sigma}(n=14)$ & $\begin{array}{c}q \\
(n=1)\end{array}$ & $\widehat{\jmath}(n=9)$ & 우 $(n=10)$ & $\hat{\sigma}(n=14)$ & ㅇ $(n=12)$ & $\widehat{\jmath}(n=21)$ & q $(n=20)$ \\
\hline Body length (mm) & 3.53 & $\begin{array}{c}2.84- \\
6.56\end{array}$ & $2.5-4.6(3.2)$ & $3.1-6.2(4.4)$ & $3.1-5.8(3.9)$ & 4.4 & $3.9-5.2(4.5)$ & $5.1-9.5(6.8)$ & $3.5-5.4(4.4)$ & $3.7-7.9(6.8)$ & $2.3-6(4.4)$ & $4.7-8.1(6.5)$ \\
\hline Body width & 140 & $\mathrm{np}$ & 70-130 (91) & 73-127 (91) & 90-200 (113) & 100 & 105-138 (117) & $105-176(131)$ & 90-160 (116) & 80-175 (127) & 65-140 (120) & 85-190 (133) \\
\hline $\begin{array}{l}\text { Cephalic vesicle } \\
\text { length }\end{array}$ & $65-70$ & $53-75$ & 35-63 (44) & 40-60 (50) & 48-70 (58) & 40 & $48-70(58)$ & 60-70 (65) & 53-75 (65) & $50-75(65.5)$ & $40-68(60)$ & $48-76(61)(n=14)$ \\
\hline $\begin{array}{l}\text { Cephalic vesicle } \\
\text { width }\end{array}$ & $33-35$ & $28-38$ & $24-31(27)$ & $23-38(31)$ & $25-45(35)$ & 32 & $28-45(34)$ & $32-40(36)$ & $27-40(33.5)$ & $28-46(38)$ & $28-40(34)$ & $\begin{array}{c}28-45(36) \\
(n=15)\end{array}$ \\
\hline Oesophagus length & 3103 & $300-360$ & $218-353(263)$ & $270-385$ (314) & 260-350 (316) & 310 & 270-330 (302) & $275-405(351)$ & $265-357$ (326.5) & 260-390 (341) & 270-355 (322) & 280-408 (344) \\
\hline Nerve ring * & np & $\mathrm{np}$ & 95-157 (115) & $117-150(130)$ & $\begin{array}{c}120-185(151) \\
(n=9)\end{array}$ & 100 & 98-200 (140) & $\begin{array}{c}103-200(154) \\
(n=7)\end{array}$ & 115-190 (154) & $\begin{array}{c}120-170(152) \\
(n=7)\end{array}$ & $\begin{array}{c}100-180(160) \\
(n=14)\end{array}$ & $\begin{array}{c}120-193(153) \\
(n=12)\end{array}$ \\
\hline Excretory pore $*$ & 250 & 180-190 & $115-320(175)$ & $\begin{array}{c}160-220(179) \\
(n=5)\end{array}$ & $172-340(246)$ & no & 148-305 (221) & $\begin{array}{c}165-301(239) \\
(n=8)\end{array}$ & $\begin{array}{c}185-307(234) \\
\quad(n=10)\end{array}$ & $\begin{array}{l}200-268(239) \\
\quad(n=5)\end{array}$ & $\begin{array}{c}190-290(254) \\
(n=12)\end{array}$ & $\begin{array}{c}220-320(257) \\
\quad(n=12)\end{array}$ \\
\hline EP/OeL (\%) & np & $\mathrm{np}$ & $52.8-90.7(65)$ & $\begin{array}{c}57.1-65.9(61.4) \\
(n=5)\end{array}$ & $61.4-101.5(77.7)$ & no & $54.2-94.1(72)$ & $\begin{array}{c}53.6-83.6(67) \\
(n=8)\end{array}$ & $60.6-79(70.1)$ & $\begin{array}{c}58.8-73.9(66.1) \\
\quad(n=5)\end{array}$ & $\begin{array}{c}61.3-85.7(77.5) \\
(n=12)\end{array}$ & $\begin{array}{c}64.1-86.1(74) \\
\quad(n=12)\end{array}$ \\
\hline Deirids * & $\mathrm{np}$ & np & $\begin{array}{c}138-235(170) \\
(n=4)\end{array}$ & 160-225 (189) & 172-340 (249) & no & $\begin{array}{c}148-240(185) \\
(n=4)\end{array}$ & $\begin{array}{c}220-298(259) \\
(n=5)\end{array}$ & $\begin{array}{c}235-327(267) \\
(n=4)\end{array}$ & $\begin{array}{c}200-268(233) \\
\quad(n=3)\end{array}$ & $\begin{array}{c}190-290(253) \\
(n=9)\end{array}$ & $\begin{array}{l}225-320(265) \\
\quad(n=10)\end{array}$ \\
\hline Spicules length & $625-660$ & - & $460-655(547)$ & - & 530-680 (585) & - & 600-710 (646) & - & 575-700 (656) & - & $550-715(652)$ & - \\
\hline SpL/BL (\%) & $\mathrm{np}$ & - & $13.6-20.8(17)$ & - & $11.7-18.1(15)$ & - & 13.1-15.7 (14) & - & $12.5-19.6(15.2)$ & - & $10.6-24.4(15.5)$ & - \\
\hline Genital cone length & $\mathrm{np}$ & - & 29-40 (33) & - & $\begin{array}{c}28-45(36) \\
(n=12)\end{array}$ & - & $35-46(38)(n=5)$ & - & $30-50(37.9)(n=7)$ & - & $35-65(43.9)(n=8)$ & - \\
\hline Gubernaculum length & h np & - & $\begin{array}{c}20-30(25) \\
\quad(n=5)\end{array}$ & - & $10-25(20)(n=5)$ & ) & $20-28(23)(n=3)$ & - & $18-25(22)(n=5)$ & - & $18-25(22.5)(n=6)$ & - \\
\hline Vulva ** & - & $200-215$ & - & 140-220 (164) & - & 150 & - & $138-253$ (186) & - & 110-252 (196) & - & $120-236(181)$ \\
\hline Vagina vera & - & np & - & 20-60 (35) & - & 16 & - & $25-43(33)(n=6)$ & - & $20-50(37)(n=8)$ & - & $10-41(25)$ \\
\hline Vestibule length & - & np & - & $\begin{array}{c}60-105(78) \\
(n=5)\end{array}$ & - & 100 & - & 97-135 (119) & - & 90-145 (120) & - & $105-150(123)$ \\
\hline $\begin{array}{l}\text { Sphincter } \\
\quad \text { length } \times \text { width }\end{array}$ & - & np & - & $\begin{array}{c}27-35(30) \times \\
34-45(39)\end{array}$ & - & $30 \times 30$ & - & $\begin{array}{c}29-38(33) \times \\
31-46(40)\end{array}$ & - & $\begin{array}{c}20-40(32) \times \\
28-44(39)\end{array}$ & - & $\begin{array}{c}25-41(39) \times \\
32-46(39)\end{array}$ \\
\hline Infundibulum length & - & np & - & $\begin{array}{c}110-168(142) \\
(n=5)\end{array}$ & - & 100 & - & $\begin{array}{c}124-185(167) \\
(n=5)\end{array}$ & - & $\begin{array}{c}100-190(145) \\
(n=2)\end{array}$ & - & $\begin{array}{c}100-182(131) \\
(n=13)\end{array}$ \\
\hline Uterus length & - & np & - & $395-1050(661)$ & - & 380 & - & $830-1600(1218)$ & - & $\begin{array}{c}735-1120(946) \\
(n=9)\end{array}$ & - & 510-1560 (1119) \\
\hline UtL/BL (\%) & - & np & - & $12.9-18.8(15.6)$ & - & 8.7 & - & $14.4-21.1(17)$ & - & $\begin{array}{c}12-19.3(14.6) \\
\quad(n=9)\end{array}$ & - & 9.4-24.2 (17) \\
\hline Tail length & - & $50-80$ & - & $40-60(47)$ & - & 50 & - & $50-85(60)$ & - & $45-85(58)$ & - & $40-65(51)$ \\
\hline Number of eggs & - & np & - & $8-25$ (13) & - & 16 & - & $16-80(46)$ & - & $11-61(30.5)$ & - & $6-69(31)$ \\
\hline Eggs length $\times$ width & - & $\begin{array}{l}50-60 \times \\
29-35\end{array}$ & - & $\begin{array}{c}55-70(61) \times \\
35-40(39) \\
(n=5)\end{array}$ & - & no & - & $\begin{array}{c}50-69(63) \times \\
30-44(36)\end{array}$ & - & $\begin{array}{c}40-66(57) \times \\
26-43(32)\end{array}$ & - & $\begin{array}{c}40-65(53) \times \\
27-40(33)\end{array}$ \\
\hline
\end{tabular}

Abbreviations: no $=$ not observed, $\mathrm{np}=$ not provided. ${ }^{*}$ Distance to apex.

** Distance to posterior extremity. 


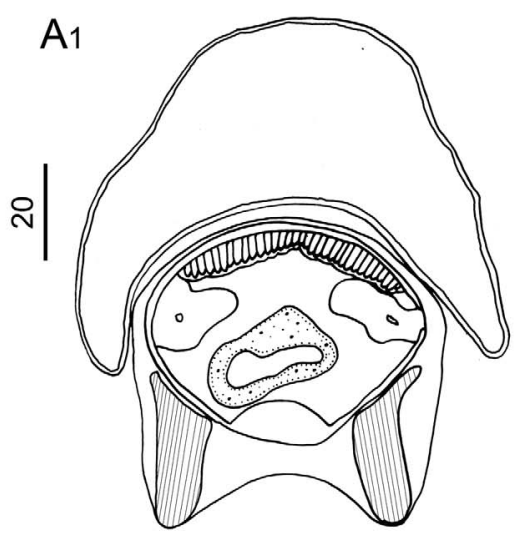

$\mathrm{B} 1$

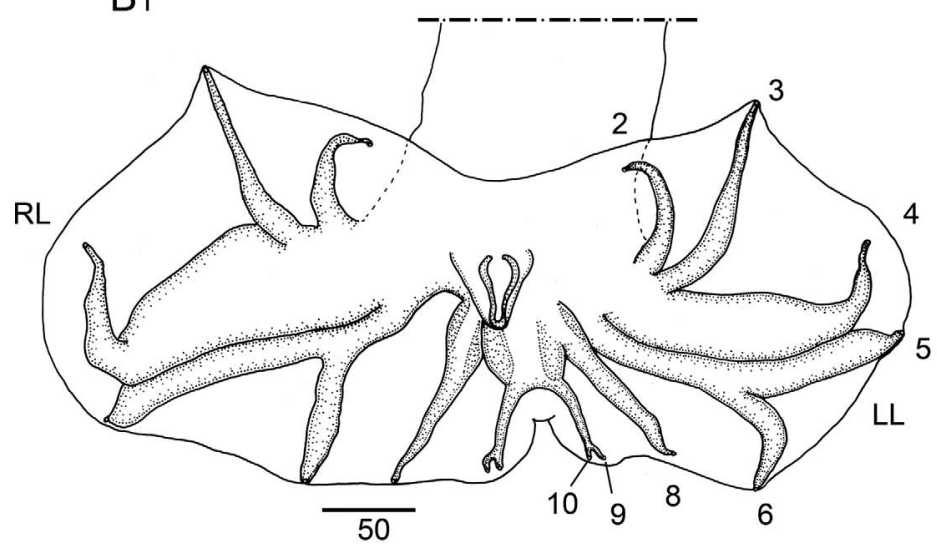

$\mathrm{B} 3$

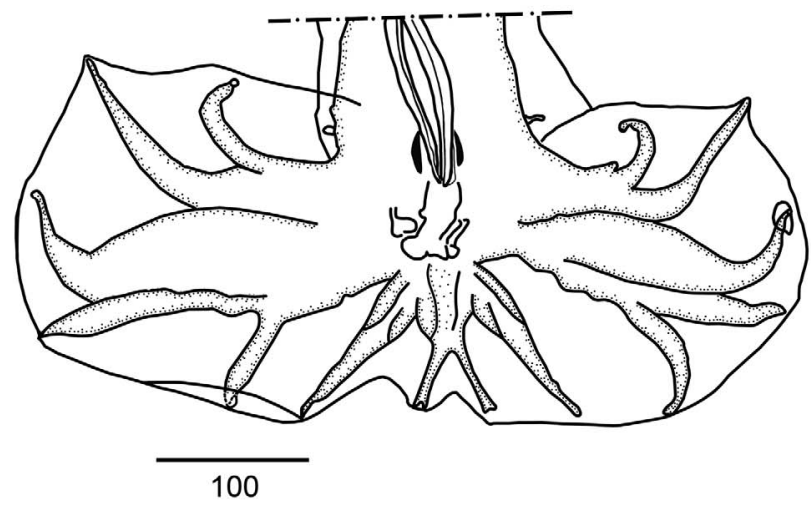

$\mathrm{A} 2$

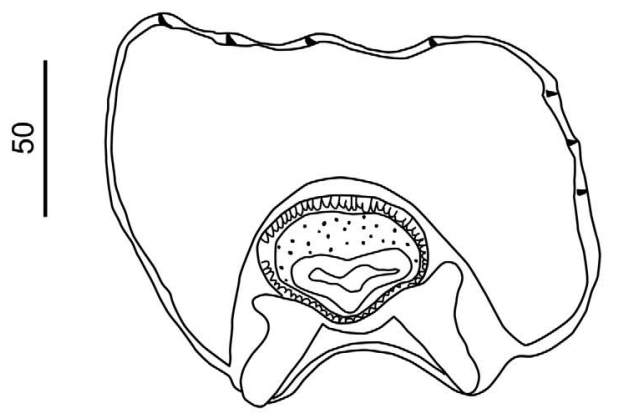

$\mathrm{B} 2$

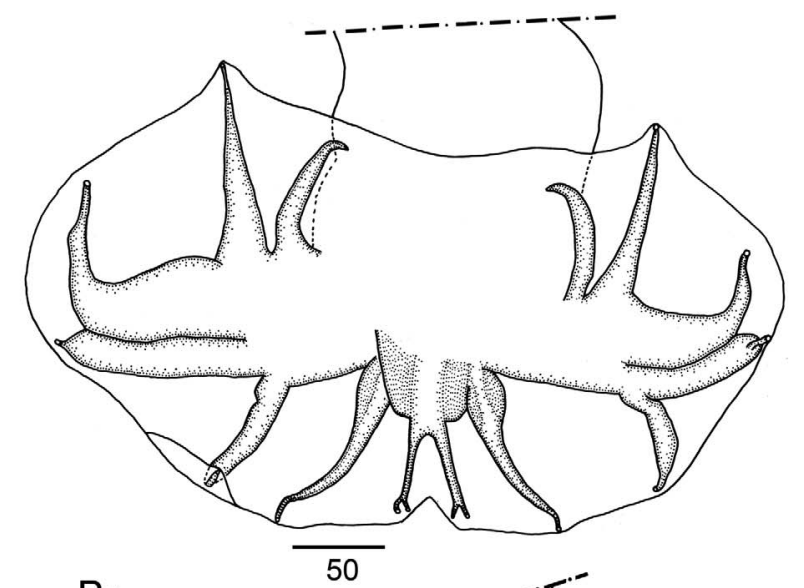

B4

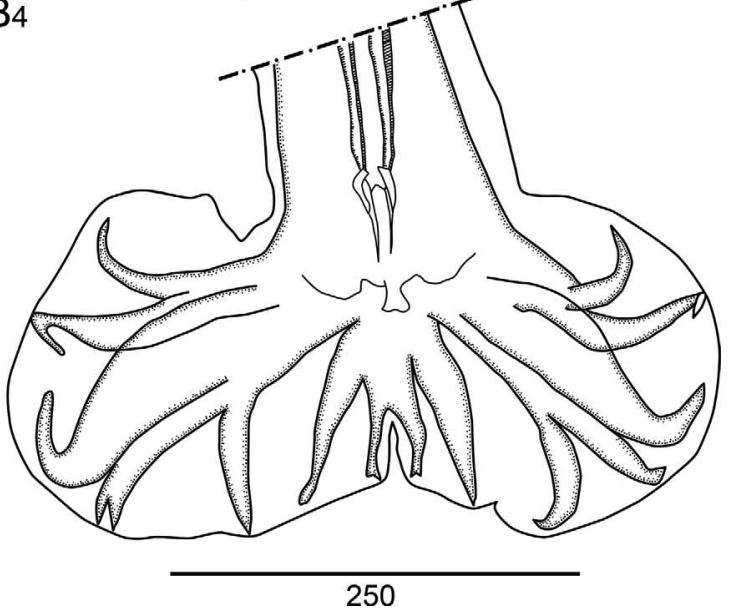

Figure 3. Hassalstrongylus dollfusi. A, female, postvulvar alae in transverse section of the body: (1) this work, (2) modified after DuretteDesset (1969). B, male, bursa, ventral views, $(1,2)$ this work, (1) with distal divergence of left ray 6, (2) with proximal divergence of left ray 6; (3) modified after Durette-Desset (1969), (4) modified after Díaz-Ungría (1963). Abbreviations: RL, right lobe, LL, left lobe.

6 of the bursa (more distal in three of our males due to a larger right lobe, see Fig. $3 \mathrm{~B}_{1}$ ), and the general aspect of the female posterior end. Related to the latter, our specimens showed, independently of the host species, different degrees of curvature and different degrees of inflation of the cuticle (Fig. 6); whereas in the type material the posterior end is curved at a right angle and the cuticular inflation ends abruptly, almost perpendicular to the body wall (Fig. $4 \mathrm{C}_{2}$ ). A degree of variability in these types of qualitative traits is expected when a large number of specimens are examined. Consequently, we identified our specimens as H. dollfusi.

Recently, Gómez-Muñoz et al. [24] reported, in two species of Oligoryzomys Bangs from Corrientes province, Argentina, specimens of nippostrongylines which were identified provisionally as Stilestrongylus sp., mainly based on characters of the synlophe. However, a more detailed study on those specimens indicated that they should be attributed to $H$. dollfusi and consequently they are included in this work. 


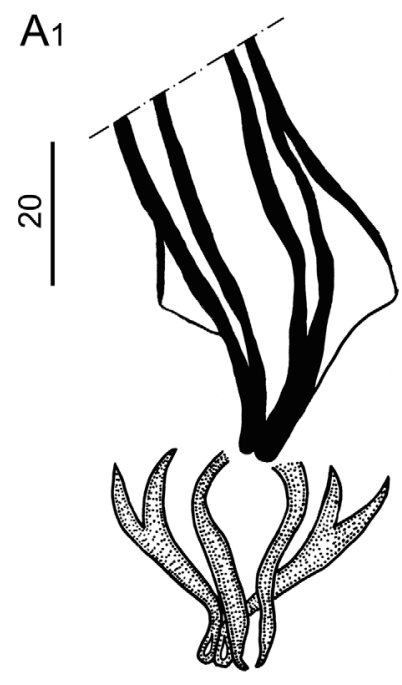

$\mathrm{A} 2$

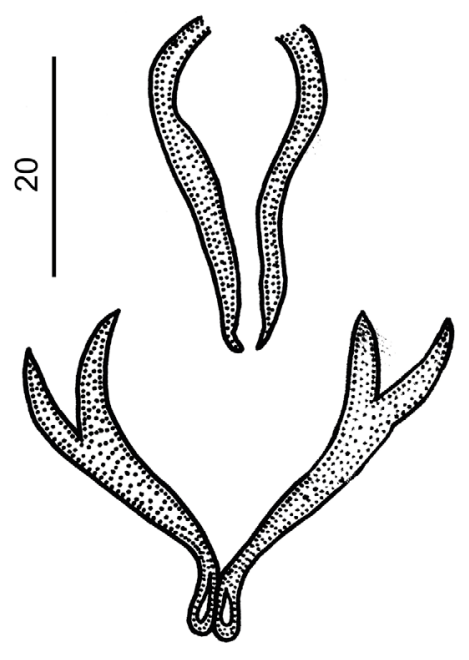

C1

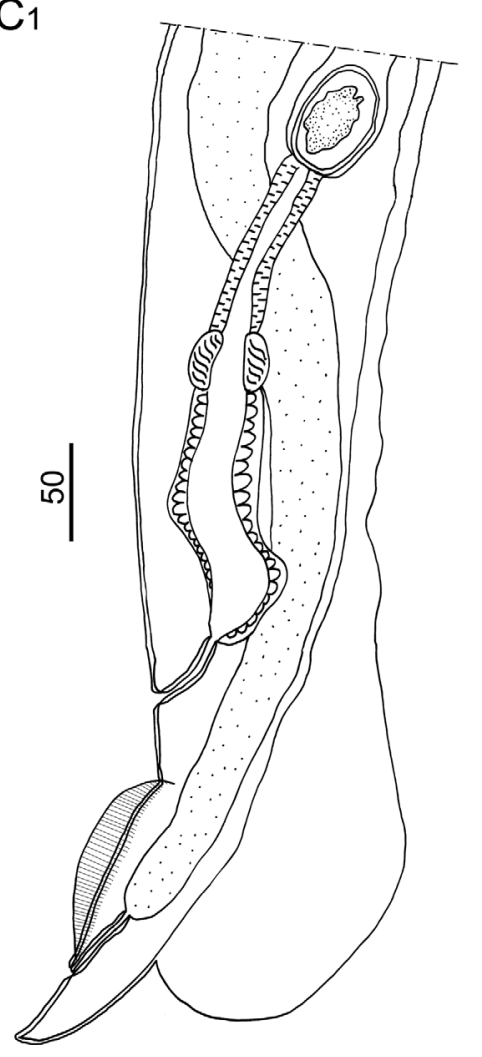

B2

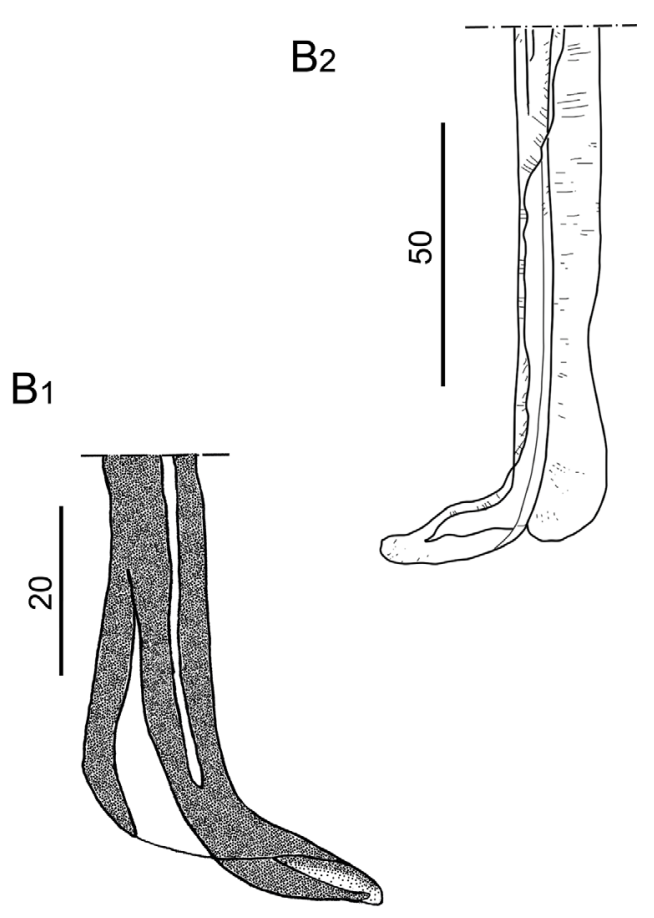

$\mathrm{C}_{2}$

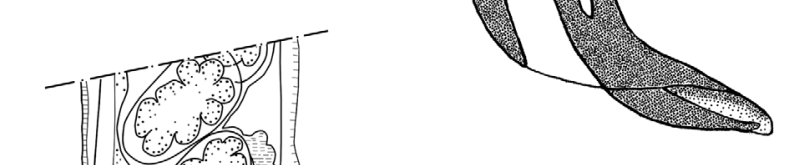

\section{B1}

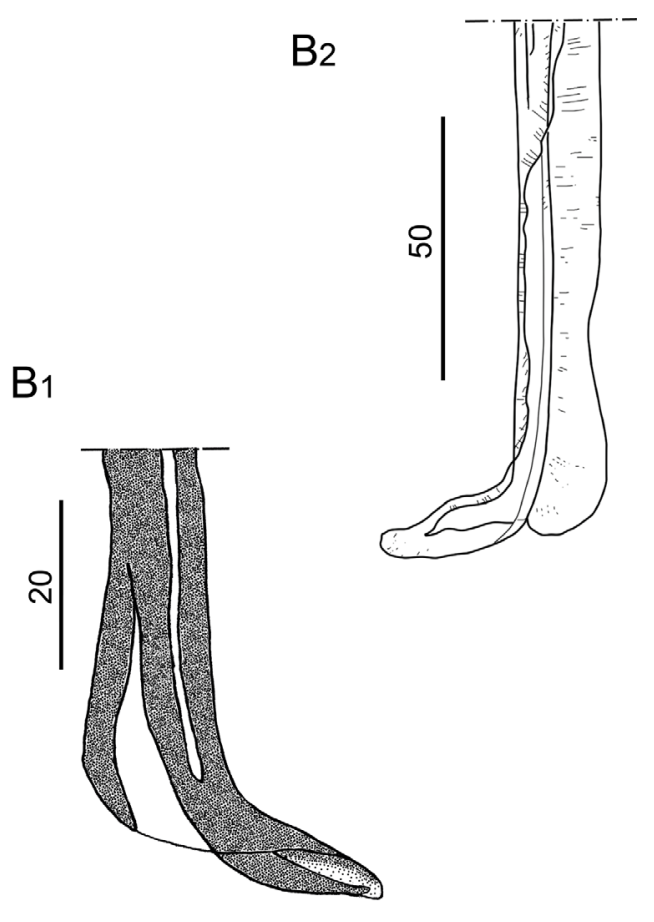

Figure 4. Hassalstrongylus dollfusi. A, B male. A, telamon: (1) ventral view with spicules tips, (2) ventral view, pieces separated. B, tip of spicule: (1) this work, (2) modified after Durette-Desset (1969). C, female posterior extremity: (1) this work, left lateral view, (2) modified after Durette-Desset (1969), right lateral view.

Values of prevalence, mean intensity and relative dominance of $H$. dollfusi (Table 1) show a strong preference of the parasite by species of Oligoryzomys, with the highest prevalence and mean intensity in $O$. fornesi (global $P=100 \%$, MI $=79.2$ ) followed by $O$. flavescens $(P=87.1 \%, \mathrm{MI}=61.1)$ and $O$. nigripes $(P=43.9 \%, M I=14)$. In contrast, in $H$. chacarius, the prevalence was lower $(P=21.6 \%)$, and the intensity extremely low $(\mathrm{MI}=2)$. In this host, the intensity of infection never exceeded 3 worms, and only one female worm was found in the whole sampling (Tables 1-3). In A. azarae, H. dollfusi was found in only one out of 28 examined hosts from the localities reported in this paper $(P=3.6 \%)$ (Fig. 1; Table 1). The highest values of relative dominance of $H$. dollfusi in the community of intestinal nippostrongylines were registered in O. flavescens and $O$. fornesi, with 98.5 and 90.8 respectively, followed by $O$. nigripes (with 37.1) and the unique A. azarae with 22.8 (Table 1). 

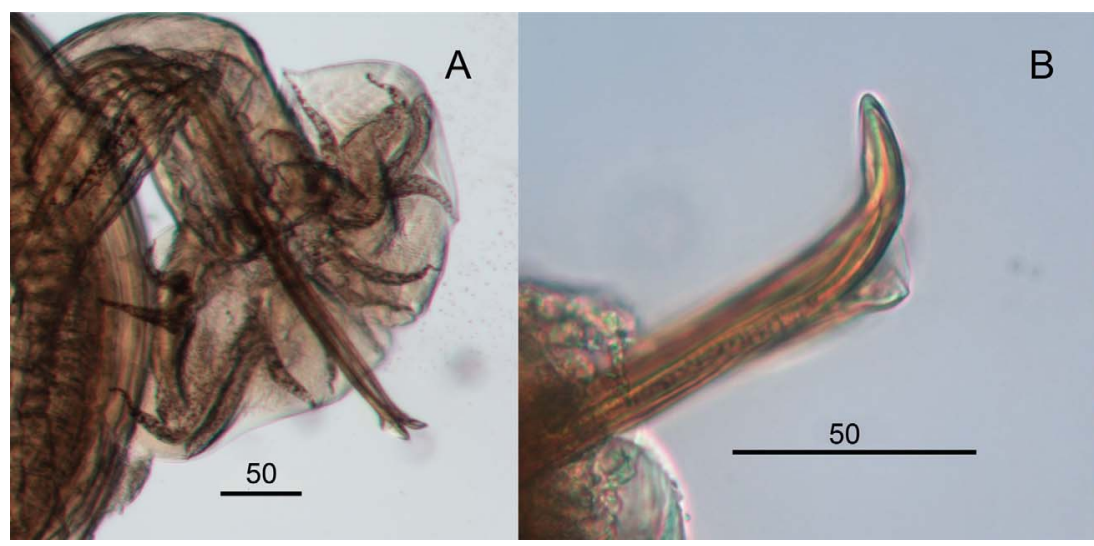

B

Figure 5. Hassalstrongylus dollfusi. Male. A, bursa, ventral view; B, C, tips of spicules, B, right lateral view, C, ventral view.

A

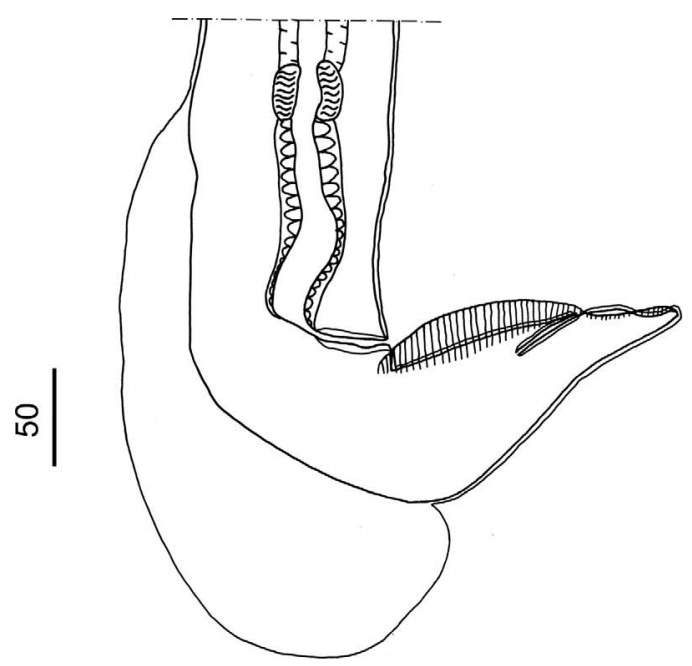

B

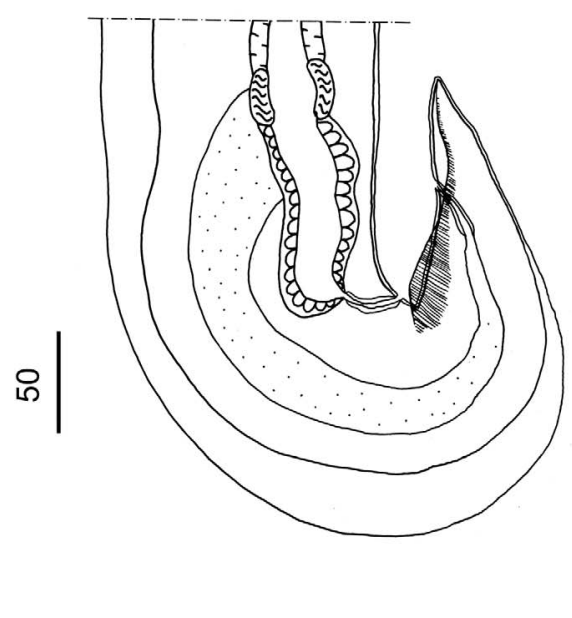

C

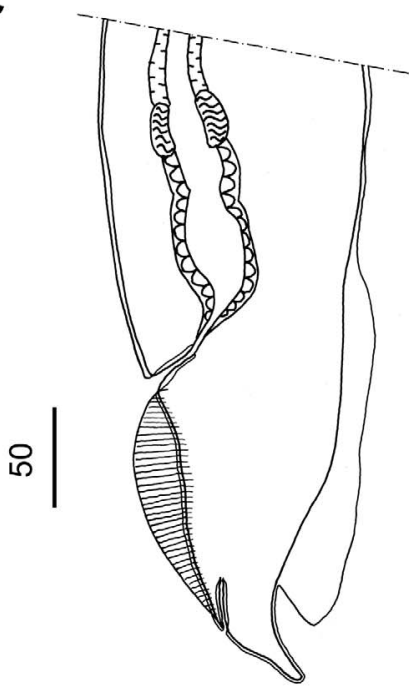

Figure 6. Hassalstrongylus dollfusi. Female, posterior extremities. A, curved ventrally at right angle, cuticular inflation ends abruptly, almost perpendicular (most specimens); B, strongly curved ventrally, cuticular inflation gradually tapering, ends obliquely; C, straight, cuticular inflation less developed. A, B, right lateral views; C, left lateral view.

\section{Discussion}

The finding of $H$. dollfusi in ten different localities in an area of about $19,000 \mathrm{~km}^{2}$, in five different host species and through several sampling events (see Table 2 for sampling details) allows us to consider $H$. dollfusi a usual component of the helminth fauna of sigmodontine rodents in this area. However, the values of prevalence and mean intensity, when considered globally by host species, greatly varied, being higher in the three species of Oligoryzomys and remarkably lower in $H$. chacarius and A. azarae (Table 1).

Data in Table 1 indicate that in the localities surveyed $O$. flavescens, O. fornesi, and $O$. nigripes act as primary hosts of $H$. dollfusi. The presence of the parasite in $H$. chacarius and A. azarae is, instead, less frequent, occurring when these latter share the habitat with the primary hosts; and even in such cases, $H$. dollfusi never appears as an important component of the helminth community. It is worth noting that in the localities reported herein, many other species of sigmodontine hosts were captured and examined for helminths. However, none of them was found to harbour $H$. dollfusi. These hosts were: Akodon montensis, Calomys callidus, C. callosus, Necromys lasiurus, N. obscurus, Oxymycyterus rufus and Sooretamys angouya.

As stated above, $H$. dollfusi was, up to now, only known from its type host Mus musculus, a non-native rodent. DíazUngría [12] remarked that the host of $H$. dollfusi was captured in the wild (forest). In this sense, it is worth noting that the type locality (Tiara, Aragua Department, Venezuela) at the time of the study was a mining settlement ( $R$. Guerrero, pers. comm.). Therefore, the presence of mice should not be surprising, and it is not unexpected that mice became infected with autochthonous nippostrongylines during incursions in the forest in the vicinities of the locality. Durette-Desset [15], when redescribing $H$. dollfusi, remarked that it was unlikely that the mouse was the primary host of the parasite, and that this latter should be found among the autochthonous cricetids. The fact that $H$. dollfusi was never reported again from mice supports this hypothesis. On the other hand, the lack of subsequent 
records of the parasite even from native hosts should be attributed probably to both the paucity of helminthological surveys and the host preference showed by the parasite.

The primary host of $H$. dollfusi should undoubtedly be an autochthonous cricetid, but which one remains a question. In South America, the genus Oligoryzomys has 19 species distributed in a continuum from north Colombia and Venezuela to extreme southern Chile and Argentina (for a detailed distribution of the Oligoryzomys species see [42]). The specimens of $H$. dollfusi studied herein, compared to those from the type locality, show remarkable morphological homogeneity. Therefore, even when the present finding is far from the type locality of $H$. dollfusi, it would not be unreasonable to suppose that the parasite could be present in an area comprising at least from the type locality in Venezuela south to northern Corrientes province in Argentina, parasitizing different species of Oligoryzomys.

However, it is also worth noting the absence of $H$. dollfusi in several other localities where populations of Oligoryzomys spp. were present and abundant. This was observed in our study (Fig. 1), but also in numerous studies carried out by Argentinian and Brazilian researchers regarding the parasitic fauna of different species of Oligoryzomys [6-8, 28, 31, 33, 38, 39].

Indeed, H. dollfusi was not found in localities south of $28^{\circ} \mathrm{S}$ (this work) where $O$. flavescens and $O$. nigripes were captured and examined for parasites (Fig. 1, localities F and H). Nor was the parasite found during an extensive study on the helminth assemblage of sigmodontine rodents (included $O$. flavescens and $O$. nigripes) from a broad wetland area of the Río de la Plata, Buenos Aires province, Argentina between $34^{\circ} 45^{\prime} \mathrm{S}, 58^{\circ} 06^{\prime} \mathrm{W}$ and $34^{\circ} 56^{\prime} \mathrm{S}, 57^{\circ} 42^{\prime} \mathrm{W}$ [31].

In a similar way, $H$. dollfusi has never been reported in any of the numerous studies on helminths of sigmodontine rodents in different localities from the Atlantic Forest from Brazil [6-8, 28, 38, 39] or Argentina [33], even when $O$. nigripes was one of the rodent species most commonly captured in such localities.

The Trichostrongylina are characterized by direct life cycles, in which the first three larval stages are free in the external environment and the stage infectious to the definitive host is the third-stage larva $[2,22]$. Therefore, the presence and persistence of the parasite in an area will be related both to the presence of populations of suitable definitive hosts and to the existence of adequate environmental conditions (temperature, humidity level, soil and vegetation type, etc.) for the free-living stages. Specific environmental requirements for the free-living stages of $H$. dollfusi are still unknown, but a probable constraint in these conditions may explain the absence of the parasite in well-surveyed populations of Oligoryzomys spp. south of $28^{\circ}$ $\mathrm{S}$ in Argentina [31] and eastward in the Argentinean and Brazilian Atlantic Forest [6-8, 28, 33, 38, 39]. The extremely low value of RD of $H$. dollfusi in the unique positive locality corresponding to the Atlantic Forest biome (PPPI, Fig. 1, Table 1) is consistent with this hypothesis.

A significant sampling effort and thorough taxonomical work in the extensive intermediate area between the type locality and the area surveyed herein would greatly improve our knowledge of hosts and geographical distribution of $H$. dollfusi.
Meanwhile, this study provides the first identification and redescription of this species in southern South America, from autochthonous hosts, and more than 50 years after its original description.

Acknowledgements. The authors would like to thank Ulyses Pardiñas and Carlos Galliari for the identification of the hosts; Marcela Lareschi, Guillermo Panisse, Juliana Sanchez and Julio Torres for their invaluable role in the collection of hosts; Natalia Guerreiro Martins, Pablo López and Soledad Paulos for their help with parasitological prospecting; Ricardo Guerrero (Universidad Central de Venezuela) for providing important information on the type host and locality of $H$. dollfusi, and Marie-Claude DuretteDesset (Muséum National d'Histoire Naturelle, Paris, France) for her constructive comments on the manuscript. Funds for this research were provided by the Agencia Nacional de Promoción de la Investigación, el Desarrollo tecnológico y la Innovación (Agencia I+D+i) through grants PICT 2019-3535, PICT 2015-1348 and PICT 2010-0924, and the Consejo Nacional de Investigaciones Cientificas y Técnicas (CONICET) through grants PIP 2014-0429 and 20100006. The authors declare that they have no conflict of interest.

\section{References}

1. Abad AD, Chávez VA, Pinedo VR, Tantaleán VM, GonzálesViera O. 2017. Helmintofauna gastrointestinal de importancia zoonótica y sus aspectos patológicos en roedores (Rattus spp) en tres medioambientes. Revista de Investigaciones Veterinarias del Perú, 27, 736-750.

2. Anderson RC. 2000. Nematode parasites of vertebrates: their development and transmission. Guilford, UK: CABI.

3. Beveridge I, Spratt DM, Durette-Desset M-C. 2014. Order Strongylida (Railliet \& Henry, 1913). Handbook of Zoology. Gastrotricha, Cycloneuralia and Gnathifera, 2, 557-612.

4. Birkenholz DE. 1963. A study of the life history and ecology of the round-tailed muskrat (Neofiber alleni True) in north-central Florida. Ecological Monographs, 33, 255-280.

5. Bush AO, Lafferty KD, Lotz JM, Shostak AW. 1997. Parasitology meets ecology on its own terms: Margolis et al. revisited. Journal of Parasitology, 83, 575-583.

6. Cardoso T dos S, Simões RO, Luque JLF, Maldonado A Jr, Gentile R. 2016. The influence of habitat fragmentation on helminth communities in rodent populations from a Brazilian Mountain Atlantic Forest. Journal of Helminthology, 90, 460.

7. Cardoso $\mathrm{T}$ dos S, Braga CA de C, Macabu CE, Simões R, Costa-Neto SF, Maldonado Júnior A, Gentile R, Luque JL. 2018. Helminth metacommunity structure of wild rodents in a preserved area of the Atlantic Forest, Southeast Brazil. Revista Brasileira de Parasitologia Veterinária, 27, 495-504.

8. Cardoso T dos S, Macabu CE, Simões R de O, Maldonado A Jr, Luque JL, Gentile R. 2019. Helminth community structure of two sigmodontine rodents in Serra dos Órgãos National Park, state of Rio de Janeiro, Brazil. Oecologia Australis, 23, 301-314.

9. Chabaud AG, Desset M-C. 1966. Nippostrongylus rauschi $\mathrm{n}$. sp. parasitic nematode of Dermoptera and considerations on $N$. brasiliensis cosmopolitan parasite of domestic rats. Annales de Parasitologie Humaine et Comparée, 41, 243-249.

10. Chandler AC. 1932. A new species of Longistriata (Nematoda) from the cotton rat, Sigmodon hispidus, with notes on the division of the heligmosominae into genera. Journal of Parasitology, 19, $25-31$. 
11. De Sotomayor CR, Serrano-Martínez E, Tantaleán VM, Quispe HM, Casas VG. 2015. Identificación de parásitos gastrointestinales en ratas de Lima Metropolitana. Revista de Investigaciones Veterinarias del Perú, 26, 273.

12. Díaz-Ungría C. 1963. Nématodes parasites, nouveaux ou intéressants, du Venezuela. Annales de Parasitologie Humaine et Comparée, 38, 893-914.

13. Digiani MC, Serrano PC, Notarnicola J, Panisse G, DuretteDesset M-C, Navone GT. 2019. Heligmonéllidos sudamericanos: ¿cuánto sabemos de su distribución hospedatoria? Revista Argentina de Parasitología, especial VIII CAP, 52.

14. Dikmans G. 1935. New nematodes of the genus Longistriata in rodents. Journal of the Washington Academy of Sciences, 25, 72-81.

15. Durette-Desset M-C. 1969. Nématodes Héligmosomes d'Amérique du Sud. IV. Description de Longistriata hoineffae n. sp., parasite de cricétidés, et étude du système des arêtes cuticulaires de L. epsilon Travassos, 1937, et L. dollfusi Díaz-Ungría, 1963. Bulletin du Muséum National d'Histoire Naturelle, 41, 328337.

16. Durette-Desset M-C. 1971. Essai de classification des Nématodes Héligmosomes. Corrélation avec la paléobiogéographie des hôtes. Mémoires du Muséum National d'Histoire Naturelle, $49,1-126$.

17. Durette-Desset M-C. 1972. Compléments morphologiques à l'étude de quelques Nématodes Héligmosomes, parasites de Rongeurs américains. Annales de Parasitologie Humaine et Comparée, 47, 243-249.

18. Durette-Desset M-C. 1985. Trichostrongyloid nematodes and their vertebrate hosts: reconstruction of the phylogeny of a parasitic group. Advances in Parasitology, 24, 239-306.

19. Durette-Desset M-C, Digiani MC. 2005. The axis of orientation of the synlophe in the Heligmosomoidea (Nematoda, Trichostrongylina): a new approach. Parasite, 12, 195-202.

20. Durette-Desset M-C, Digiani MC. 2005. Systematic position of some Nearctic Heligmosomoidea (Nematoda: Trichostrongylina) from the US National Parasite Collection and their description. Journal of Parasitology, 91, 893-899.

21. Durette-Desset M-C, Digiani MC. 2012. The caudal bursa in the Heligmonellidae (Nematoda: Trichostrongylina). Characterization and hypothesis on its evolution. Parasite, 19, 3.

22. Durette-Desset M-C, Digiani MC, Kilani M, Geffard-Kuriyama D. 2017. Critical revision of the Heligmonellidae (Nematoda: Trichostrongylina: Heligmosomoidea). París: Mémoires du Muséum National d'Histoire Naturelle.

23. Garcia-Prieto L, Falcon-Ordaz J, Guzman-Cornejo C. 2012. Helminth parasites of wild Mexican mammals: list of species, hosts and geographical distribution. Zootaxa, 3290, 1-92.

24. Gómez-Muñoz M de los A, Robles M del R, Milano MF, Digiani MC, Notarnicola J, Galliari C, Navone GT. 2020. Helminths from Sigmodontinae rodents (Muroidea: Cricetidae) in Humid Chaco ecoregion (Argentina): a list of species, host and geographical distribution. Revista Mexicana de Biodiversidad, 25, 221-227.

25. Grandón-Ojeda A, Moreno L, Landaeta C. 2017. Prevalencia de endoparásitos de Rattus rattus en Chile. Parasitología Latinoamericana, 66, 368.

26. Hancke D, Navone G, Suarez O. 2011. Endoparasite community of Rattus norvegicus captured in a shantytown of Buenos Aires City, Argentina. Helminthologia, 48, 167-173.
27. Hancke D, Suárez OV. 2018. Structure of parasite communities in urban environments: the case of helminths in synanthropic rodents. Folia Parasitologica, 65, 1-9.

28. Kersul MG, Costa NA, Boullosa RG, Silva AA, Rios ÉO, Munhoz AD, Andrade-Silva BE, Júnior AM, Gentile R, Alvarez MR. 2020. Helminth communities of sigmonontine rodents in cocoa agroforestry systems in Brazil. International Journal for Parasitology: Parasites and Wildlife, 11, 62-71.

29. Kinsella JM. 1988. Comparison of helminths of Rice Rats, Oryzomys palustris, from freshwater and saltwater marshes in Florida. Proceedings of the Helminthological Society of Washington, 55, 275-280.

30. Landaeta-Aqueveque C, Robles M del R, Henríquez A, YáñezMeza A, Correa JP, González-Acuña D, Cattan PE. 2018. Phylogenetic and ecological factors affecting the sharing of helminths between native and introduced rodents in Central Chile. Parasitology, 145, 1570-1576.

31. Navone GT, Notarnicola J, Nava S, Robles M del R, Galliari C, Lareschi M. 2009. Arthropods and helminths assemblage in sigmodontine rodents from wetlands of the Río de la Plata, Argentina. Mastozoología Neotropical, 16, 121-133.

32. Panisse G. 2015. Patrones de diversidad de helmintos en roedores sigmodontinos de la Selva Atlántica Interior en Argentina, PhD Thesis, Universidad Nacional de La Plata.

33. Panisse G, Robles M del R, Digiani MC, Notarnicola J, Galliari C, Navone GT. 2017. Description of the helminth communities of sympatric rodents (Muroidea: Cricetidae) from the Atlantic Forest in northeastern Argentina. Zootaxa, 4337, 243-262.

34. Panti-May JA, Hernández-Betancourt SF, Rodríguez-Vivas RI, Robles M del R. 2015. Infection levels of intestinal helminths in two commensal rodent species from rural households in Yucatán, Mexico. Journal of Helminthology, 89, 42.

35. Panti-May JA, Digiani MC, Palomo-Arjona EE, GurubelGonzález YM, Navone GT, Williams CM, HernándezBetancourt SF, Robles M del R. 2018. A checklist of the helminth parasites of sympatric rodents from two Mayan villages in Yucatán, México. Zootaxa, 4403, 495.

36. Patton JL. 2015. Suborder Myomorpha Brants, 1855, in Mammals of South America. Patton JL, Pardiñas UF, D'Elía G, Editors. University of Chicago Press: Chicago, Illinois. p. 58-60.

37. QGIS Association. 2021. QGIS Geographic Information System.

38. Simões RO, Maldonado-Júnior A, Luque JL. 2012. Helminth communities in three sympatric rodents from the Brazilian Atlantic Forest: contrasting biomass and numerical abundance. Brazilian Journal of Biology, 72, 909-914.

39. Simões RO, Souza JGR, Maldonado A Jr, Luque JL. 2011. Variation in the helminth community structure of three sympatric sigmodontine rodents from the coastal Atlantic Forest of Rio de Janeiro, Brazil. Journal of Helminthology, 85, 171.

40. Travassos L. 1914. Contribuições para o conhecimento da fauna helmintolojica brazileira: III. Memórias do Instituto Oswaldo Cruz, 6, 150-162.

41. Underwood HT, Owen JG, Engstrom MD. 1986. Endohelminths of three species of Oryzomys (Rodentia: Cricetidae) from San Luis Potosí, Mexico. Southwestern Naturalist, 31, 410-411.

42. Weksler M, Bonvicino CR. 2015. Genus Oligoryzomys Bangs, 1900, in Mammals of South America. Patton JL, Pardiñas UF, D’Elía G, Editors. University of Chicago Press: Chicago, Illinois. p. 417-437.

43. Wilson DE, Reeder DM. 2005. Mammal species of the world: a taxonomic and geographic reference. Baltimore, Maryland: JHU Press.

Cite this article as: Serrano PC, Digiani MC, Gómez-Muñoz M de los A, Notarnicola J, Robles M del R \& Navone GT. 2021. Hassalstrongylus dollfusi (Nematoda, Heligmonellidae): rediscovery in native South American rodents, six decades after its description. Parasite 28, 80. 
An international open-access, peer-reviewed, online journal publishing high quality papers on all aspects of human and animal parasitology

Reviews, articles and short notes may be submitted. Fields include, but are not limited to: general, medical and veterinary parasitology; morphology, including ultrastructure; parasite systematics, including entomology, acarology, helminthology and protistology, and molecular analyses; molecular biology and biochemistry; immunology of parasitic diseases; host-parasite relationships; ecology and life history of parasites; epidemiology; therapeutics; new diagnostic tools.

All papers in Parasite are published in English. Manuscripts should have a broad interest and must not have been published or submitted elsewhere. No limit is imposed on the length of manuscripts.

Parasite (open-access) continues Parasite (print and online editions, 1994-2012) and Annales de Parasitologie Humaine et Comparée (1923-1993) and is the official journal of the Société Française de Parasitologie. 